\title{
A Decline in Aminotransferase Activity Due to Lifestyle Modification in Children with NAFLD
}

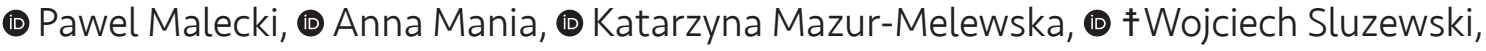 \\ (1) Magdalena Figlerowicz
}

Poznan University of Medical Sciences, Department of Infectious Diseases and Child Neurology, Poznan, Poland

‡: We are terribly sorry to announce that Professor Wojciech Sluzewski passed away on the date of 19 September 2020. We thank him for his contributions.

\begin{abstract}
Aim: As obesity rates in children and adolescents increase, non-alcoholic fatty liver disease (NAFLD) has become the most common liver disease affecting this organ. This study aimed to evaluate the effectiveness of lifestyle modifications as a method of improving liver function indexes in children with NAFLD.

Materials and Methods: The study group consisted of 49 patients with NAFLD (35 boys and 14 girls), age range 3-16 years (mean $10.51 \pm 3.18$ years). Anthropometric measurements were taken and laboratory tests were performed. Features of steatosis of the liver were evaluated by ultrasound. The mean follow-up period was $2.45 \pm 1.45$ years. Aspartate aminotransferase (AST) to [Platelet (PLT) Ratio and Pediatric NAFLD Fibrosis Score (PNFS)] were calculated. The Mediterranean diet and physical exercises were recommended. End-point parameters were a decrease in the aminotransferase level and body weight.

Results: In the study group, 35 children (71.43\%) were obese, and 7 (14.29\%) were overweight. In 22/49 cases (44.9\%) body mass index (BMI) loss was achieved, in the other patients, BMI gain was noted. Abnormal alanine aminotransferase (ALT) levels were observed in 25/49 children. In the group of children with initial increased ALT levels, significant reduction of ALT, AST and gamma-glutamyltransferase (CGT) levels were found with regard to baseline values. There was also a significant decline in mean AST to PLT Ratio index (APRI) level and PLT count. Moreover, a decrease in ALT level was negatively related to PNFS, APRI and GGT.

Conclusion: Lifestyle modification leads to a significant decrease in aminotransferase levels, even in children who fail to achieve BMI reduction. Keywords: Fatty liver, obesity, children, aminotransferases
\end{abstract}

\section{Introduction}

Non-alcoholic fatty liver disease (NAFLD) is currently the most common chronic liver illness in children (1). This term describes a broad spectrum of liver disorders, ranging from simple steatosis to non-alcoholic steatohepatitis. Its estimated prevalence ranges from $3 \%$ to $12 \%$ in the general pediatric population and up to $70 \%$ in obese children (2). Suspicion of liver steatosis is usually based on imaging studies - a characteristic, hyperechogenic picture of the liver in abdominal ultrasound is the most commonly used mode of assessment. Diagnosis is established by excluding other causes of liver damage (drug poisoning, viral hepatitis, autoimmune liver disease, inborn errors of metabolism, alcoholism) (3,4).

With regard to the increase in the incidence of NAFLD, also in the pediatric population, and the possible consequences caused by its complications, the search for treatment methods has become the subject of many scientific studies. 
According to the guidelines created recently by two medical societies, namely the North American Society for Pediatric Gastroenterology, Hepatology and Nutrition and the American Association for the Study of Liver Diseases (AASLD), lifestyle modification remains the primary method of treatment. Currently, there are no clear guidelines which present precise recommendations on lifestyle changes, such as diet type or specific weight loss. Recommendations are extrapolated from studies on the adult population. In everyday clinical practice, the first step in both the diagnosis and assessment of disease progression is to determine aminotransferases and abdominal ultrasound.

Liver fibrosis is an independent factor increasing the risk of liver-related complications and mortality (5). Liver biopsy remains a golden although imperfect standard when steatohepatitis or liver fibrosis is suspected. Furthermore, the procedure should be performed in the case of uncertain diagnosis, possible overlap syndromes or additional risk factors such as type 2 diabetes. Nevertheless, since liver biopsy is an invasive procedure with its own bias, initial assessment of fibrosis and inflammation of the liver tissue is usually performed using non-invasive tools such as serum markers or panels of markers (6). Non-invasive evaluation of fibrosis has been the subject of research of scientists for several years. Panels based on basic laboratory tests and anthropometric measurements [e.g. Aspartate aminotransferase (AST) to [Platelet (PLT) Ratio index (APRI)], Pediatric NAFLD Fibrosis score (PNFS)] are used as well as and more extensive ones, requiring more specific tests (e.g. cytokeratin 18, enhanced liver fibrosis). Serum markers enable the repetitive assessment and therefore, are used as a tool for the monitoring of the treatment $(7,8)$.

The aim of this study was to evaluate aminotransferase level and liver damage indexes with regard to the decline in body weight as a result of lifestyle modifications in children with NAFLD.

\section{Materials and Methods}

The group in our prospective, consecutive study consisted of 35 boys and 14 girls, with a diagnosis of NAFLD, age range 3-16 years (mean 10.51 \pm 3.18 years), who visited the Hepatology Outpatient Clinic at the Department of Infectious Diseases and Child Neurology. The sample size was determined using "Sample Size Calculator" (https:// clincalc.com/stats/samplesize.aspx).

The diagnosis of NAFLD was based on medical history, physical examination and additional tests-increased aminotransferases level and hepatic steatosis in abdominal ultrasound. The characteristics for NAFLD abnormalities in the liver ultrasound (hyperechogenicity, poor visualization of the diaphragm, intrahepatic vessels and posterior part of the right lobe using Samsung HS40 machine) were the inclusion criteria of this study (9). In 3 cases, according to clinical indications (uncertain diagnosis, suspected significant liver injury based on laboratory tests), a liver biopsy was performed using the Menghini technique. In differential diagnosis, viral hepatitis (type B, C, Epstein-Barr virus and cytomegalovirus infection), autoimmune hepatitis, deficiency of alfa-1-antitrypsin, hemochromatosis, celiac disease, Wilson disease, cystic fibrosis, thyroid disease, drug intake and alcohol intake were excluded. Furthermore, in children younger than 5 years, the presence of inborn errors of metabolism such as fatty acid oxidation disorders, galactosemia and tyrosinemia were excluded $(1,10)$. Children with the diagnosed conditions mentioned above were excluded from the study group. None of the described patients had diabetes. The baseline characteristics of the research group are included in Table I.

The weight of the patients was assessed on an electronic digital scale (Radwag WPT 60/150 O; $150 \mathrm{~kg}$ capacity and 10 g accuracy), height was measured with a $2 \mathrm{~m}$ vertical wall stadiometer graduated in millimeters (Charder HM200PW). We performed measurements following recommended procedures. Patients were classified in the obese group based on body mass index (BMI) depending on age [BMI=weight/ (height) ${ }^{2}\left(\mathrm{~kg} / \mathrm{m}^{2}\right)$ ] greater than 2 standard deviations (SD) above the World Health Organization (WHO) Growth Reference median and in the overweight group when BMIfor-age was more significant than $1 \mathrm{SD}$ above the WHO Growth Reference median (11).

Complete blood count, alanine aminotransferase (ALT), AST, gamma-glutamyltransferase (GCT) and serum lipids were assessed on standard laboratory analyzers. Normal upper values for liver enzymes were as follows: ALT $<12$ yo: boys $<40 \mathrm{IU} / \mathrm{L}$, girls $<35 \mathrm{IU} / \mathrm{L}$; $>12$ yo: boys $<26 \mathrm{IU} / \mathrm{L}$, girls $<22$ IU/L, AST <12 yo: $<45 \mathrm{IU} / \mathrm{L},>12$ yo: boys $<29 \mathrm{IU} / \mathrm{L}$, girls $<25$ IU/L, GGT 4-24 IU/L.

The Homeostatic Model Assessment of Insulin Resistance (HOMA-IR) was calculated according to the following formula:

HOMA-IR=(insulin $(\mathrm{mU} / \mathrm{mL})^{*}$ glucose $(\mathrm{mmol} / \mathrm{L}) / 22.5$. The result should not exceed 1

\section{Non-invasive Hepatic Fibrosis Scores}

APRI and PNFS were calculated based on established formulas $(8,12)$. 
APRI = AST/ULN (upper limit of normal)/PLTs (G/L)*100. APRI $>1.0$ has a sensitivity of $76 \%$ and specificity of $72 \%$ for predicting cirrhosis. APRI $>0.7$ has a sensitivity of $77 \%$ and specificity of $72 \%$ for predicting significant hepatic fibrosis. The lower the APRI score $(<0.5)$, the greater the negative predictive value and the ability to rule in cirrhosis.

PNFS (probability distribution) $=\left[\frac{\mathrm{e}^{\mathrm{z}}}{1+\mathrm{e}^{\mathrm{z}}}\right] * 100 ; \mathrm{z}=1.1+(0.34 * \sqrt{ } \mathrm{ALT})$ $+\left(0.002^{*} A L P\right)-\left[1.1^{*} \log (P L T\right.$ G/L) $]-\left(0.02^{*} \mathrm{CGT}\right)$

\section{Intervention and Control Visits}

Before enrollment to the study, ALT and AST levels were evaluated at least 3 times with an interval of 3 to 6 months. Enrolled patients were recommended to modify their lifestyle: moderate aerobic physical exercise (at least 60 minutes 5 times a week), for example, brisk walking, cycling, swimming and additionally the Mediterranean diet - 5 portions of vegetables and fruits per day, olive oil, higher fish intake (at least 2-3 portions per week), cereals or grains and dairy products (milk, yoghurt) for breakfast, nuts (at least 2-3 portions per week), pulses (at least 1 portion per week), pasta or rice almost daily, and also a reduction in red meat, simple sugars and saturated fat. It was recommended to avoid sugar and sugar-containing beverages. It was also recommended to use computers/other screen equipment or watch TV for less than 2 hours a day. Weight reduction was not recommended in children with BMI within reference values. However, lifestyle modification was introduced in this group of children to reduce ultrasound abnormalities in the liver. Compliance to all parts of lifestyle intervention was carefully evaluated during every visit based on medical history, interviews with the children and their caregivers as well as the analysis of the weekly menu diary. All children were followed every three months during the first year of the follow-up and every 6 months afterwards during outpatient visits. The result as a note "adheres to/does not comply with lifestyle modification principles" in the medical records was prepared. Body mass and height measurements were made, and blood samples were taken during every visit according to clinical indications. During the check-up visits, their weekly menu was analyzed, if it met the minimum criteria described above, "adheres to lifestyle modification principles" was marked. The restriction modifications were semi-quantified: red meat less than twice a week, elimination of fast-food and sweets, drinking water instead of sweet drinks. The physical level was assessed according to the principles described above. The mean follow-up period was $2.45 \pm 1.45$ years.

Table I. Baseline group characteristics

\begin{tabular}{|c|c|c|c|c|c|}
\hline Parameter & Number & Percentage & Mean \pm SD & Median & Range \\
\hline Age (years) & - & - & $10.51 \pm 3.18$ & 11 & $3-16$ \\
\hline Sex: male/female & $35 / 14$ & $71.4 / 28.6$ & - & - & - \\
\hline Weight (kg) & - & - & $68.23 \pm 25.1$ & 62.5 & $24.5-122.5$ \\
\hline Height (cm) & - & - & $153.42 \pm 18.27$ & 153 & $114.5-190$ \\
\hline BMI & - & - & $28.08 \pm 5.84$ & 27.51 & $15.43-42.13$ \\
\hline $\mathrm{BMI}>+2 \mathrm{SD}$ & 35 & 71.43 & - & - & - \\
\hline$+1 \mathrm{SD}<\mathrm{BMI} \leq+2 \mathrm{SD}$ & 7 & 14.29 & - & - & - \\
\hline $\mathrm{BMI} \leq+1 \mathrm{SD}$ & 7 & 14.29 & - & - & - \\
\hline WHR & - & - & $0.94 \pm 0.07$ & 0.93 & $0.82-1.06$ \\
\hline $\operatorname{ALT}(I U / L)$ & - & & $61.35 \pm 57.54$ & 42.5 & $12-304$ \\
\hline AST (IU/L) & - & - & $46.21 \pm 28.65$ & 35.5 & $19-149$ \\
\hline GGT (IU/L) & - & - & $26.83 \pm 17.96$ & 21 & $9-75$ \\
\hline Total cholesterol (mg/dL) & - & - & $166.39 \pm 40.29$ & 162 & $56-249$ \\
\hline LDL (mg/dL) & - & - & $99.07 \pm 32.72$ & 101.5 & $18-151$ \\
\hline $\mathrm{HDL}(\mathrm{mg} / \mathrm{dL})$ & - & - & $46.56 \pm 21.99$ & 41 & $25-148$ \\
\hline $\mathrm{TG}(\mathrm{mg} / \mathrm{dL})$ & - & - & $137.85 \pm 62.05$ & 126.5 & $23-265$ \\
\hline HOMA-IR & - & - & $4.82 \pm 4.64$ & 3.65 & $1.4-23.1$ \\
\hline
\end{tabular}

SD: Standard deviation, BMI: Body mass index, WHR: Waist to hip ratio, ALT: Alanine aminotransferase, AST: Aspartate aminotransferase, GGT: Gammaglutamyltransferase, LDL: Low density lipoprotein, HDL: High density lipoprotein, TG: Triglycerides, HOMA-IR: Homeostatic model assessment-insulin resistance 


\section{Statistical Analysis}

Statistical analysis was made with STATISTICA (version 13, TIBCO Software Inc., Palo Alto, USA). Data were presented as the mean \pm SD. Mann-Whitney $U$ or Student t-tests were used to compare continuous variables, where appropriate. Pearson chi-square test was applied to categorical variables. Kruskal-Wallis test was used for testing whether samples originate from the same distribution. Wilcoxon-Signed rank test was used to evaluate dependent parameters. We used Fisher's Exact test in the analysis of contingency tables. Correlations were performed using the Spearman rank correlation test. $\mathrm{P}<0.05$ was considered statistically significant.

The study design was approved by the Bioethics Committee of the Poznan University of Medical Sciences (no: 1074/17 of $9^{\text {th }}$ November 2017). Informed consent was obtained from each patient included in the study. The study protocol follows the ethical guidelines of the 1975 Declaration of Helsinki as reflected in a priori approval by the institution's human research committee.

\section{Results}

Boys comprised the majority of the study group (35/49, $71.43 \%)$. In the whole study group, 35 children (71.43\%) were obese, 7 (14.29\%) were overweight, and 7 (14.29\%) had normal weight. All patients were advised to modify their lifestyle according to described suggestions. In 22/49 cases (44.9\%) BMI reduction was achieved (at least 10\% from the baseline), in the remaining ones (27/49, 55.1\%) BMI increased compared to initial values. In the study group, patients who followed lifestyle modification advice were compared to the group who did not follow the regimen. The latter group was considered as a comparative group. Among patients with a decrease in BMI, 100\% complied with the recommendations for lifestyle changes. In the group with an increase in BMI, all of these patients were described as "does not comply with the lifestyle modification principles". This group included patients who did not meet at least 2 dietary and/or physical level criteria.

The comparison of children with and without observed $\mathrm{BMI}$ reduction does not include those children with normal initial body weight. In the group of patients with BMI reduction, significantly lower levels of ALT, GGT and APRI at the endpoint were found. In spite of the lack of weight reduction, a decline in ALT level was also observed in the latter group. A comparison of both groups is provided in Table II.

Abnormal ALT level was observed in 25/49 children. In the group of children with increased ALT level, significant reduction of ALT, AST and GGT level was found with regard to baseline values (Table III). There was also a significant decline in mean APRI level and PLT count.

A statistically significant decrease in ALT and GGT level was found in the group with a reduction in BMI (respectively $p=0.0028$ and $p=0.044$, Figure 1). Furthermore, in the group with increased initial ALT, higher initial APRI values $(0.52 \pm 0.28$ vs. $0.29 \pm 0.20 ; p=0.002)$ were observed (Table III).

The decline in ALT level was negatively related to PNFS $(r=-0.61 ; p<0.05$; Figure 2a), APRI $(r=-0.45 ; p<0.05$; Figure $2 b)$ and GGT level $(r=-0.41 ; p<0.05$; Figure $2 c)$.

Table II. Comparison of clinical parameters in patients with and without BMI reduction

\begin{tabular}{|c|c|c|c|c|c|c|}
\hline \multirow[b]{2}{*}{ Parameter } & \multicolumn{3}{|c|}{ Patients with BMI reduction $n=22$} & \multicolumn{3}{|c|}{ Patients without $B M I$ reduction $n=20$} \\
\hline & Baseline & End-point & $\mathbf{p}$ & Baseline & End-point & $\mathbf{p}$ \\
\hline Gender M/F & \multicolumn{3}{|l|}{$15 / 7$} & \multicolumn{2}{|l|}{$13 / 7$} & 0.827 \\
\hline Weight (kg) & $74.07 \pm 25.92$ & $76.83 \pm 19.85$ & 0.313 & $68.58 \pm 22.83$ & $82.78 \pm 26.86$ & $0.0006^{*}$ \\
\hline Height (cm) & $151.42 \pm 17.5$ & $163.07 \pm 14.2$ & $0.00005^{*}$ & $155.03 \pm 19.67$ & $163.64 \pm 19.39$ & $0.006^{*}$ \\
\hline BMI $\left(\mathrm{kg} / \mathrm{m}^{2}\right)$ & $31.31 \pm 4.81$ & $28.48 \pm 4.43$ & $0.0007^{*}$ & $27.69 \pm 3.73$ & $30.06 \pm 4.08$ & $0.00004^{*}$ \\
\hline ALT (IU/L) & $65.47 \pm 60.39$ & $38.71 \pm 45.12$ & $0.0282^{*}$ & $53.44 \pm 39.85$ & $42.81 \pm 21.21$ & 0.227 \\
\hline AST (IU/L) & $46.65 \pm 30.86$ & $35.0 \pm 34.59$ & 0.092 & $41.5 \pm 20.96$ & $33.75 \pm 13.39$ & 0.126 \\
\hline GGT (IU/L) & $34.92 \pm 25.36$ & $25.85 \pm 16.35$ & $0.044^{*}$ & $21.71 \pm 9.2$ & $21.64 \pm 6.72$ & 0.97 \\
\hline PLT (G/L) & $282.24 \pm 67.99$ & $272.76 \pm 85.25$ & 0.397 & $299.5 \pm 66.0$ & $278.56 \pm 59.31$ & 0.079 \\
\hline APRI & $0.42 \pm 0.27$ & $0.31 \pm 0.17$ & $0.037^{*}$ & $0.36 \pm 0.18$ & $0.31 \pm 0.11$ & 0.217 \\
\hline
\end{tabular}




\section{Discussion}

According to the well-known regularity, NAFLD affects the vast majority of children with excessive body mass (13). In the described study group, all children were Caucasians. Most of them were boys, which aligns with previously published works (14). Suspicion of NAFLD in children is usually based on increased level of aminotransferases, especially ALT. According to studies, in obese and overweight children, elevated liver function tests have a sensitivity of $88 \%$ and specificity of $26 \%(15)$. In the study population, just over half of the children (51\%) had slightly elevated liver function tests at baseline.
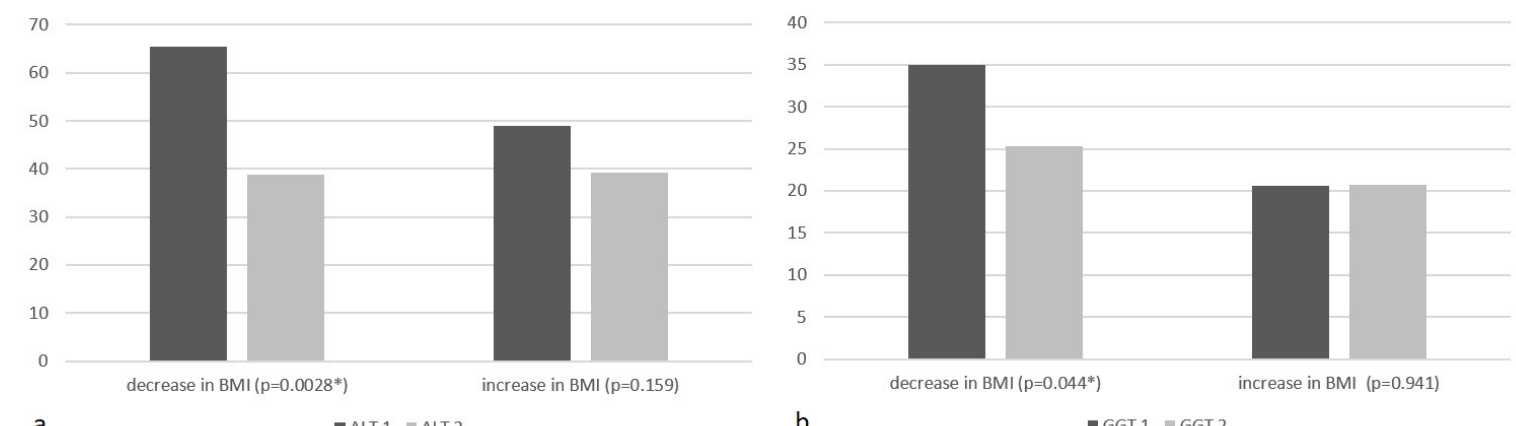

Figure 1. Comparison of ALT (a) and GGT (b) level in the group with and without a decrease in BMI at the beginning and the end of the observation period

ALT: Alanine aminotransferase, GGT: Gamma-glutamyltransferase, BMI: Body mass index
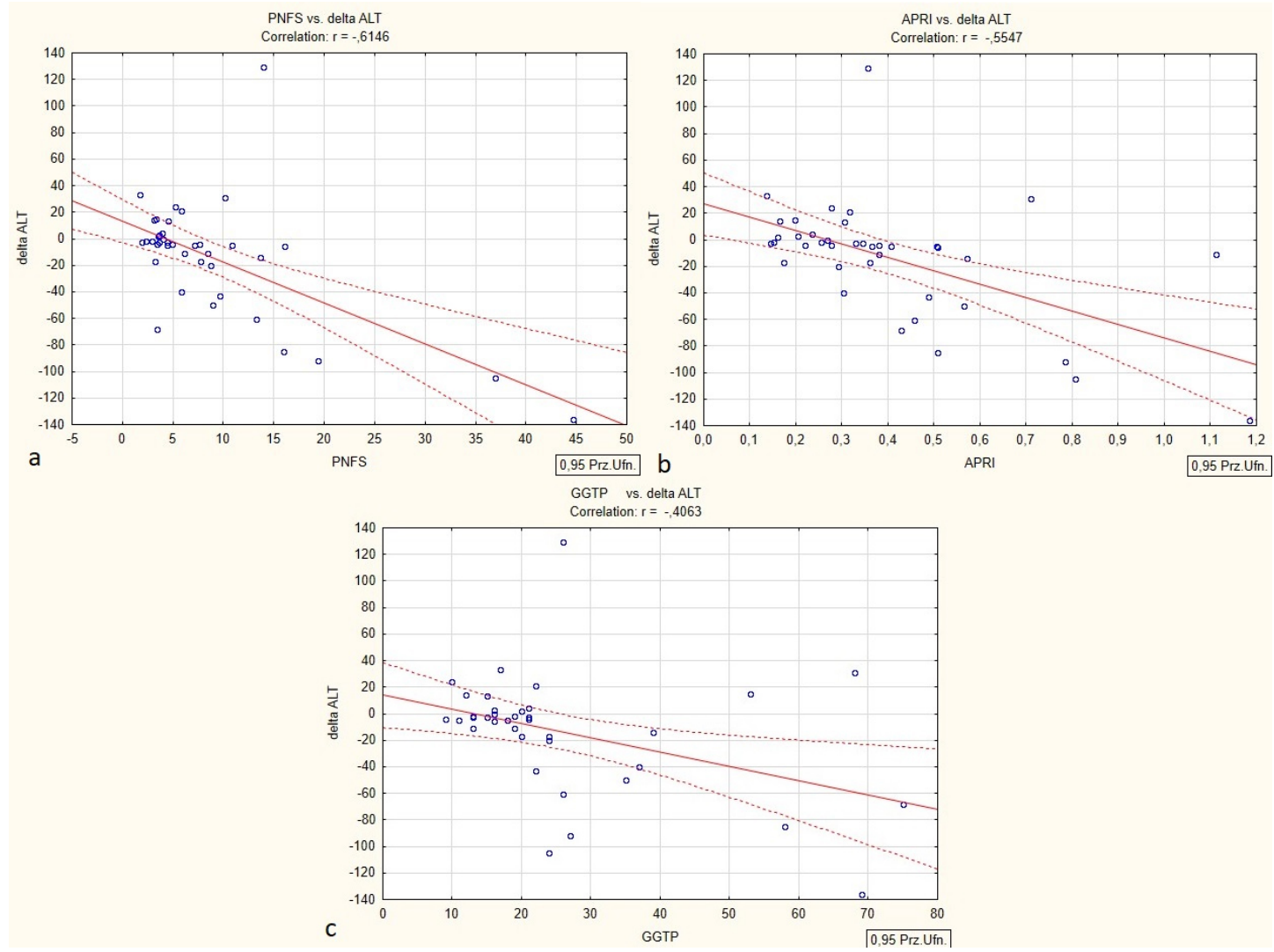

Figure 2. Correlation between the decrease in ALT level and PNFS (a), APRI (b), and GGT level (c)

ALT: Alanine aminotransferase, PNFS: Pediatric NAFLD fibrosis score, APRI: Aspartate aminotransferase to platelet ratio index, GGT: Gammaglutamyltransferase 
Table III. Comparison of clinical parameters in children regarding ALT level at baseline and at the end of the follow-up period

\begin{tabular}{|c|c|c|c|c|c|c|}
\hline \multirow[b]{2}{*}{ Parameter } & \multicolumn{3}{|c|}{$\begin{array}{l}\text { Patients with increased ALT activity at baseline } \\
n=25\end{array}$} & \multicolumn{3}{|c|}{$\begin{array}{l}\text { Patients with normal ALT activity at baseline } \\
\mathrm{n}=\mathbf{2 4}\end{array}$} \\
\hline & Baseline & End-point & p & Baseline & End-point & p \\
\hline Gender M/F & \multicolumn{3}{|l|}{$19 / 6$} & \multicolumn{2}{|l|}{$16 / 8$} & 0.153 \\
\hline Weight (kg) & $71.67 \pm 22.64$ & $78.26 \pm 23.99$ & $0.008^{*}$ & $64.48 \pm 27.74$ & $71.65 \pm 25.28$ & $0.003^{*}$ \\
\hline Height $(\mathrm{cm})$ & $156.52 \pm 16.47$ & $164.86 \pm 16.43$ & $0.0009^{*}$ & $150.30 \pm 20.28$ & $158.38 \pm 17.26$ & $0.0001^{*}$ \\
\hline BMI $\left(\mathrm{kg} / \mathrm{m}^{2}\right)$ & $28.69 \pm 5.55$ & $28.17 \pm 5.46$ & 0.619 & $27.31 \pm 6.25$ & $27.72 \pm 5.24$ & 0.476 \\
\hline ALT (IU/L) & $86.96 \pm 45.66$ & $59.79 \pm 52.86$ & $0.027^{*}$ & $24.09 \pm 6.99$ & $26.57 \pm 12.57$ & 0.768 \\
\hline AST (IU/L) & $58.00 \pm 25.85$ & $42.00 \pm 32.67$ & $0.0023^{*}$ & $29.43 \pm 10.80$ & $27.57 \pm 5.76$ & 0.387 \\
\hline GGT (IU/L) & $36.33 \pm 19.54$ & $28.28 \pm 12.80$ & $0.008^{*}$ & $14.75 \pm 3.51$ & $17.13 \pm 4.65$ & 0.059 \\
\hline PLT (G/L) & $297.44 \pm 71.43$ & $286.04 \pm 78.91$ & $0.022^{*}$ & $281.70 \pm 74.25$ & $264.09 \pm 63.46$ & $0.029 *$ \\
\hline APRI & $0.52 \pm 0.28$ & $0.36 \pm 0.18$ & $0.004^{*}$ & $0.29 \pm 0.20$ & $0.27 \pm 0.08$ & 0.903 \\
\hline
\end{tabular}

BMI: Body mass index, ALT: Alanine aminotransferase, AST: Aspartate aminotransferase, GGT: Gamma-glutamyltransferase, APRI: AST to Platelet Ratio index, PLT: Platelet

Lifestyle modification plays a crucial role in the treatment of patients with NAFLD. Weight reduction of around $10 \%$ of the initial values is usually associated with an improvement in the markers of liver injury $(16,17)$. The value of ALT decline as a marker of histological improvement, and thus, the effectiveness of NAFLD treatment has been confirmed in the past (18). In our research group, due to the ongoing growth of our patients, weight reduction was not observed. However, patients with a decrease in BMI at the end of the observation showed significantly lower ALT levels. Furthermore, even partial compliance to lifestyle modification without weight reduction resulted in a decline in ALT level.

The Mediterranean diet is usually considered as a recommended nutritional intervention in children and adults (19-22). In our study, this type of diet was recommended, resulting in a reduction of $\mathrm{BMI}$ and an improvement in biochemical parameters in a significant proportion of described patients (19). Compliance with dietary recommendations and lifestyle changes were assessed by the parents as being quite challenging to implement in their children's lives. Due to the inconvenience and fear of poor cooperation, the complete quantitative assessment of diet compliance (e.g. weighing portions) was abandoned. The medical history and the general analysis of the menu were considered a reliable source of information.

Given the observed association of ALT level with a decrease in BMI in the research group, the AASLD recommendations for biannual screening with serum ALT in obese children and $\mathrm{BMI}$ in the $85^{\text {th }}-94^{\text {th }}$ percentile and other risk factors seem to be appropriate $(3,23)$.
Elevated BMI is an independent factor that increases the risk of liver fibrosis. According to the studies of lacobellis et al. (24), the significant upper limit of BMI above which the risk of fibrosis increases is 26.3 [odds ratio: $5.85,95 \%$ confidence interval $(\mathrm{Cl})$ : 1.6-21]. In the analysis of the presented research group, a decrease in ALT level was negatively related to PNFS and APRI. The PNFS index has an area under the receiver operating characteristic (AUROC) value of 0.74 for advanced fibrosis, the highest among non-invasive fibrosis indicators tested in children so far. Therefore, its relation to improving the health of patients who underwent lifestyle modification was emphasized.

Out of AST/ALT ratio, APRI, PNFS, and FIB-4 fibrosis scores as markers of hepatic fibrosis in children with NAFLD, APRI was characterized by high efficacy in the diagnosis without distinguishing the advancement of the process (25). In the results of our research, the negative correlation between the decrease in ALT level and the APRI value was statistically significant. Higher initial values of parameters indicating liver injury are not a factor reducing the effectiveness of lifestyle modifications in improving the clinical status of children with NAFLD. Interestingly, lower PLT count at the end of the observation period was observed in the group of patients with ALT decline. The reason for the higher initial PLT count may be chronic inflammation and oxidative stress resulting from obesity or iron deficiency in this group of patients (26).

A negative correlation between the decrease in ALT level and GGT was found in our study. According to previous reports, advanced fibrosis can be correlated with high GGT level. The level of this enzyme $>45 \mathrm{IU} / \mathrm{L}$ had AUROC 0.65 
(95\% Cl 0.5-0.8) (27). A statistically significant reduction in GCT in the ALT and BMI decrease groups may confirm the clinical utility of this parameter. The correlation between high CGT level and insulin resistance (IR) was also described. Additionally, the relation between IR and advanced fibrosis was observed only in patients with elevated CGT (28).

\section{Study Limitations}

The limitations of this study include the lack of a control group of overweight children without NAFLD undergoing lifestyle modification. It was also challenging to assess patient involvement in diet and exercise recommendations. The analysis did not include the histopathological assessment of liver tissue due to the small number of patients who underwent liver biopsy.

\section{Conclusions}

Lifestyle modification has a significant effect on the decrease in aminotransferase levels, even in those children who fail to achieve weight loss.

Changes in diet and physical exercise have a positive effect on improving the parameters of liver function regardless of their pre-lifestyle modification values.

\section{Ethics}

Ethics Committee Approval: The study design was approved by the Bioethics Committee of the Poznan University of Medical Sciences (no: 1074/17 of 9th November 2017).

Informed Consent: Informed consent was obtained from each patient included in the study.

Peer-review: Externally and internally peer-reviewed.

\section{Authorship Contributions}

Concept: P.M., A.M., K.M.M., W.S., M.F., Design: P.M., A.M., Data Collection or Processing: P.M., Analysis or Interpretation: P.M., A.M., M.F., Literature Search: P.M., W.S., Writing: P.M., M.F.

Conflict of Interest: None of the authors had conflict of interest.

Financial Disclosure: The authors declared that this study received no financial support.

\section{References}

1. Vajro P, Lenta S, Socha P, et al. Diagnosis of nonalcoholic fatty liver disease in children and adolescents: position paper of the ESPGHAN Hepatology Committee. I Pediatr Gastroenterol Nutr 2012; 54:700-13.

2. Nobili V, Alisi A, Newton KP, Schwimmer JB. Comparison of the phenotype and approach to pediatric vs. adult patients with nonalcoholic fatty liver disease. Gastroenterology 2016; 150:1798-810.

3. Chalasani N, Younossi Z, Lavine JE, et al. The diagnosis and management of nonalcoholic fatty liver disease: Practice guidance from the American Association for the Study of Liver Diseases. Hepatology 2017; 67:328-57.

4. Alfani R, Vassallo E, De Anseris AG, et al. Pediatric fatty liver and obesity: not always just a matter of nonalcoholic fatty liver disease. Children (Basel) 2018; 5:169.

5. Ekstedt $M$, Hagstrom $H$, Nasr $P$, et al. Fibrosis stage is the strongest predictor for disease-specific mortality in NAFLD after up to 33 years of follow-up. Hepatology 2015; 61:1547-54.

6. Flisiak-Jackiewicz M, Lebensztejn DM. Update on pathogenesis, diagnostics and therapy of nonalcoholic fatty liver disease in children. Clin Exp Hepatol 2019; 5:11-21.

7. Mandelia C, Kabbany MN, Conjeevaram Selvakumar PK, Alkhouri $\mathrm{N}$. The search for noninvasive methods to identify liver fibrosis in children with nonalcoholic fatty liver disease. Biomark Med 2018; 12:265-73.

8. Alkhouri N, Mansoor S, Giammaria P, Liccardo D, Lopez R, Nobili $\mathrm{V}$. The development of pediatric NAFLD fibrosis score (PNFS) to predict the presence of advanced fibrosis in children with nonalcoholic fatty liver disease. PLoS One 2014; 9:e104558.

9. Ballestri S, Romagnoli D, Nascimbeni F, Francica G, Lonardo A. Role of ultrasound in the diagnosis and treatment of nonalcoholic fatty liver disease and its complications Expert Rev Gastroenterol Hepatol 2015; 9:603-27.

10. Shah J, Okubote T, Alkhouri N. Overview of Updated practice guidelines for pediatric nonalcoholic fatty liver disease. Gastroenterol Hepatol (NY) 2018; 14:407-14

11. World Health Organization. Obesity and overweight. Geneva: World Health Organization 2018. Last Accessed Date: 01.11.2019 Available from: https://www.who.int/news-room/fact-sheets/ detail/obesity-and-overweight

12. Chou R, Wasson N. Blood tests to diagnose fibrosis or cirrhosis in patients with chronic hepatitis $C$ virus infection: a systematic review. Ann Intern Med 2013; 158:807-20.

13. Schwimmer JB, Deutsch R, Kahen T, Lavine JE, Stanley C, Behling.C. Prevalence of fatty liver in children and adolescents. Pediatrics 2006; 118:1388-93.

14. Anderson EL, Howe LD, Jones HE, Higgins IPT, Lawlor DA, Fraser A. The prevalence of nonalcoholic fatty liver disease in children and adolescents: a systematic review and meta-analysis. PLoS One 2015; 10:e0140908.

15. Schwimmer JB, Newton KP, Awai $\mathrm{HI}$, et al. Paediatric gastroenterology evaluation of overweight and obese children referred from primary care for suspected nonalcoholic fatty liver disease. Aliment Pharmacol Ther 2013; 38:1267-77.

16. Koot BG, Van der Baan-Slootweg OH, Tamminga-Smeulders $\mathrm{CL}$. Lifestyle intervention for nonalcoholic fatty liver disease: prospective cohort study of its efficacy and factors related to improvement. Arch Dis Child 2011; 96:669-74.

17. Vos M, Abrams S, Barlow S, et al. NASPGHAN Clinical Practice Guideline for the Diagnosis and Treatment of Nonalcoholic Fatty Liver Disease in Children: Recommendations from the Expert Committee on NAFLD (ECON) and the North American Society of Pediatric Gastroenterology, Hepatology and Nutrition (NASPGHAN). J Pediatr Gastroenterol Nutr 2017; 64:319-34. 
18. Lavine JE, Schwimmer JB, Van Natta ML, et al. Effect of vitamin E or metformin for treatment of nonalcoholic fatty liver disease in children and adolescents: the TONIC randomized controlled trial. JAMA 2011; 305:1659-68.

19. De Ruyter JC, Olthof MR, Seidell JC, Katan MB. A trial of sugarfree or sugar-sweetened beverages and body weight in children. N Engl I Med 2012; 367:1397-40.

20. Ryan MC, Itsiopoulos C, Thodis T, et al. The Mediterranean diet improves hepatic steatosis and insulin sensitivity in individuals with non-alcoholic fatty liver disease. J Hepatol 2013; 59:138-43.

21. Cakir M, Akbulut UE, Okten, A. Association between Adherence to the Mediterranean Diet and Presence of Nonalcoholic Fatty Liver Disease in Children. Childhood Obesity 2016; 12:279-85.

22. St-Jules DE, Watters CA, Brunt EM, et al. Nonalcoholic Steatohepatitis Clinical Research Network. Estimation of fish and-3 fatty acid intake in pediatric nonalcoholic fatty liver disease. J Pediatr Gastroenterol Nutr 2013; 57:627-33.

23. Barlow SE, Expert Committee. Expert committee recommendations regarding the prevention, assessment, and treatment of child and adolescent overweight and obesity: summary report. Pediatrics 2007; 120:164-92.
24. lacobellis A, Marcellini M, Andriulli A, et al. Non-invasive evaluation of liver fibrosis in paediatric patients with nonalcoholic steatohepatitis. World / Gastroenterol 2006; 12:7821-5.

25. Mansoor S, Yerian L, Kohli R, et al. The evaluation of hepatic fibrosis scores in children with nonalcoholic fatty liver disease. Dig Dis Sci 2015; 60:1440-7.

26. Vuong J, Qiu Y, La M, Clarke G, Swinkels DW, Cembrowski G. Reference intervals of complete blood count constituents are highly correlated to waist circumference: Should obese patients have their own "normal values?" Am J Hematol 2014; 89:671-67.

27. Petta S, Macaluso FS, Barcellona MR, et al. Serum gammaglutamyl transferase levels, insulin resistance and liver fibrosis in patients with chronic liver diseases. PLoS One 2012; 7:e51165.

28. Shukla A, Kapileswar S, Gogtay N, et al. Simple biochemical parameters and a novel score correlate with absence of fibrosis in patients with nonalcoholic fatty liver disease. Indian । Gastroenterol 2015; 34:281-5. 